\title{
Performance of the ASTRI Mini-Array at the Observatorio
} del Teide

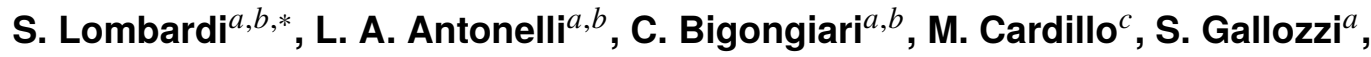
J. G. Green ${ }^{a, b}$, F. Lucarelli ${ }^{a, b}$, F. G. Saturni ${ }^{a, b}$ for the ASTRI Project ${ }^{d}$

a INAF - Osservatorio Astronomico di Roma, Via Frascati 33, 00078 Monte Porzio Catone (Rome), Italy

${ }^{b}$ ASI Space Science Data Center, Viale del Politecnico s.n.c., 00133 Rome, Italy

${ }^{c}$ INAF - Istituto di Astrofisica e Planetologia Spaziale di Roma, Via Fosso del Cavaliere 100, 00133 Rome, Italy

${ }^{d}$ http://www.astri.inaf.it/en/library/

E-mail: saverio.lombardi@inaf.it

The ASTRI Mini-Array (ASTRI MA) is a project led by the Italian Istituto Nazionale di Astrofisica (INAF) to build and operate a next-generation imaging atmospheric Cherenkov telescope observatory for ground-based gamma-ray astronomy in the energy range between $1 \mathrm{TeV}$ and $100 \mathrm{TeV}$ and beyond. It will be composed of nine small-sized ( $\sim 4$ meter in diameter) and large field-of-view ( $\sim 10$ degrees) double-mirror telescopes equipped with silicon photo-multiplier cameras. The full array will be operational within the next few years at the Observatorio del Teide (Tenerife, Spain). It will allow deep observations of the galactic and extragalactic sky with a significantly improved performance at multi-TeV energies compared to current arrays of Cherenkov telescopes, providing a fully functional complement to both present- and next-generation gamma-ray observatories in the Northern Hemisphere. In order to assess the performance of the system and to generate suitable Instrument Response Functions for high-level scientific studies, detailed high-statistic Monte Carlo simulations were generated and subsequently processed with A-SciSoft (ASTRI Scientific Software), the scientific software package of the ASTRI Project. In this contribution, we describe the main features of both the simulation and data processing chains and present the performance of the ASTRI MA achieved with the aforementioned Monte Carlo simulations.

$37^{\text {th }}$ International Cosmic Ray Conference (ICRC 2021)

July 12th - 23rd, 2021

Online - Berlin, Germany

\footnotetext{
*Presenter
} 


\section{Introduction}

Very high-energy (VHE, E $>O(100) \mathrm{GeV}$ ) gamma rays of cosmic origin can reveal unique information on open issues of modern astrophysics, particle physics, and cosmology [1]. Additionally, they also provide an important probe for multi-wavelength and multi-messenger astronomy.

Astrophysical sources of VHE photons can be observed from the ground with different types of techniques and instruments [2]. Among them, the imaging atmospheric Cherenkov telescope (IACT) technique is aimed to efficiently image extended air showers generated by astrophysical gamma rays and cosmic rays in the Earth's atmosphere through the detection of faint flashes of Cherenkov light emitted by the secondary particles produced in the cascades. The present-generation IACTs, which include H.E.S.S., MAGIC, and VERITAS, have been pursuing VHE astrophysics and fundamental physics programs for almost two decades, in synergy, among others, with the space-borne telescopes Fermi-LAT and AGILE, and the water Cherenkov detector array HAWC [3].

Gamma-ray astronomy is entering a new era thanks to a new generation of ground-based observatories, especially the future Cherenkov Telescope Array Observatory (CTAO) [4]. The CTAO is designed to be the largest and most sensitive gamma-ray observatory from a few tens of $\mathrm{GeV}$ up to the multi-TeV energy band [5]. It will consist of two arrays of Cherenkov telescopes, one in each of Earth's hemispheres, and is expected to begin science operations at full capacity within this decade. Complementary to the CTAO, other next-generation ground-based facilities will be capable of exploring the gamma-ray sky with unprecedented performance, particularly in the multi$\mathrm{TeV}$ energy band. These include the Large High Altitude Air Shower Observatory (LHAASO) [6], the future Southern Wide-field Gamma-ray Observatory (SWGO) [7], and the ASTRI Mini-Array (ASTRI MA) [8].

The ASTRI MA is under construction at the Observatorio del Teide (in the Canary Island of Tenerife, Spain), in collaboration with the Spanish Instituto de Astrofísica de Canarias (IAC), and will be fully operational within the next few years. It will be composed of nine small-sized ( $\sim 4$ meter in diameter) and large field-of-view ( $\sim 10$ degrees) double-mirror IACTs equipped with silicon photo-multiplier cameras managed by a fast front-end electronics. The design of the ASTRI MA telescopes is an evolution of that of the ASTRI-Horn telescope [9], a prototype developed by INAF within the CTA Project currently operating on Mt. Etna (Italy). The ASTRI MA will represent an important facility for deep observations of galactic and extragalactic targets at the $\mathrm{TeV}$ and multi-TeV energy scale [10], in synergy with the present- (VERITAS [11], MAGIC [12], HAWC [13]) and next-generation (LHAASO [6], CTAO Northern Array [5]) gamma-ray facilities in the Northern Hemisphere. In this contribution, we present the main performance of the system derived from detailed Monte Carlo (MC) simulations.

\section{Monte Carlo simulations}

The performance of the ASTRI MA presented in this contribution was obtained from a dedicated MC production (dubbed ASTRI MA Prod2-Teide). Air showers initiated by gamma rays, protons and electrons were simulated using the CORSIKA package [14] (version 6.99), while the response of the array telescopes was simulated using the sim_telarray package [15] (version 2018-11-07). The simulation of the dual-mirror optics, Cherenkov camera, trigger logic, and readout system of the 
ASTRI telescopes were properly implemented in the sim_telarray code [16] and cross-checked against a custom code. Simulations were performed exploiting distributed computing, namely the European Grid Infrastructure and the DIRAC framework [17].

The layout of the ASTRI MA telescopes adopted in the MC simulations is shown in Fig. 1 (left panel) and represents the most up-to-date (as for July 2021) telescope positions at the Teide Observatory site $\left(28.30^{\circ} \mathrm{N}, 16.51^{\circ} \mathrm{W}, 2390 \mathrm{~m}\right.$ a.s.l. $)$. The inter-telescope distances, defined as the distances of each telescope to its nearest neighbour, are also shown in Fig. 1 (right panel). The nominal telescope pointing configuration, in which all telescopes point to the same sky position, was assumed in all MC simulations. Air showers produced by the primaries were simulated as coming from a zenith angle of $20^{\circ}$ and an azimuth angle of $0^{\circ}$ and $180^{\circ}$ (corresponding to pointings toward the geomagnetic ${ }^{1}$ North and South, respectively). Although not-negligible differences in performance were found between the two azimuthal pointings, all results presented in this contribution were obtained by averaging between the two directions. Finally, all MC simulations were generated with a night sky background level corresponding to dark sky conditions at the Teide Observatory site [18]. The most relevant shower simulation parameters, as well as the total number of simulated events for each primary particle species, are summarized in Tab. 1.
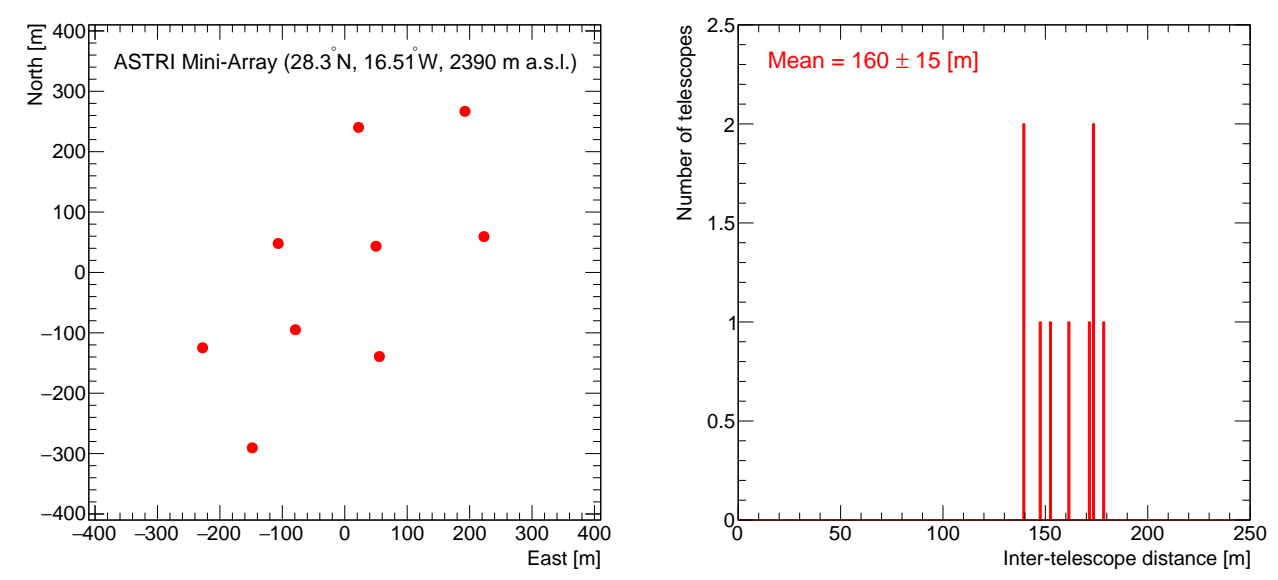

Figure 1: Left: Layout of the ASTRI MA telescopes (red circles) at the Teide Observatory site. Right: Inter-telescope distances, defined as the distances of each telescope to its nearest neighbour.

\section{Data reduction and analysis}

The MC simulations described in Sec. 2 were reduced and analyzed with A-SciSoft [19] (version 0.3.1), the scientific software package of the ASTRI Project. The software is designed to handle both real and MC data from the raw level up to the generation of scientific products. It comprises a set of independent modules, efficiently wrapped in pipelines, that perform the complete chain of data reduction and analysis. The scientific products are obtained by means of either specifically developed science tools (included in the software package) or external ones, such as the ctools [20] and Gammapy [21]. The software has been extensively checked on a MC basis, in both

\footnotetext{
${ }^{1}$ The local geomagnetic field at the Teide Observatory site was properly accounted for in the MC simulations.
} 


\begin{tabular}{cccccccc}
\hline $\begin{array}{c}\text { particle type } \\
\text { (particle component) }\end{array}$ & $\begin{array}{c}\text { spectral } \\
\text { slope }\end{array}$ & $\begin{array}{c}\text { energy } \\
\text { range } \\
{[\mathrm{TeV}]}\end{array}$ & $\begin{array}{c}\text { view cone } \\
\text { radius } \\
{[\mathrm{deg}]}\end{array}$ & $\begin{array}{c}\text { scatter } \\
\text { radius } \\
{[\mathrm{m}]}\end{array}$ & $\begin{array}{c}\text { azimuth } \\
\text { angle } \\
{[\mathrm{deg}]}\end{array}$ & $\begin{array}{c}\text { zenith } \\
\text { angle } \\
{[\mathrm{deg}]}\end{array}$ & $\begin{array}{c}\text { total number of } \\
\text { simulated showers }\end{array}$ \\
\hline \hline gamma (point-like) & -1.5 & $0.1-330$ & 0 & 2000 & $0 / 180$ & 20 & $4 \cdot 10^{7}$ \\
\hline gamma (diffuse) & -1.5 & $0.1-330$ & 10 & 2400 & $0 / 180$ & 20 & $4 \cdot 10^{8}$ \\
\hline electron & -1.5 & $0.1-330$ & 10 & 2400 & $0 / 180$ & 20 & $2 \cdot 10^{8}$ \\
\hline proton & -1.5 & $0.1-600$ & 10 & 2400 & $0 / 180$ & 20 & $2 \cdot 10^{9}$ \\
\hline \hline
\end{tabular}

Table 1: Parameters describing the MC air shower simulations used to estimate the performance of the ASTRI MA at the Teide Observatory site presented in this contribution.

single-telescope and array modes, and successfully exploited for the detection of the Crab Nebula with the ASTRI-Horn telescope [9]. A-SciSoft is currently being adopted for the reduction and analysis of the ASTRI-Horn data, the assessment of the ASTRI MA performance, and the generation of the Instrument Response Functions (IRFs) for high-level scientific studies [10]. The software is under further development in order to implement new high-performance methods for the event reconstruction and to enable the complete reduction and analysis of the upcoming real data acquired with the ASTRI MA.

The calibration and reconstruction of the MC events were achieved with the standard methods implemented in the data reduction pipeline (see [19] for more details). In particular, the background rejection and energy reconstruction were achieved with a procedure based on the Random Forest method [22], while the arrival direction of each shower was estimated from the intersection of the major axes of the images from different telescopes. After the full reconstruction of the MC events, the background (proton and electron) and gamma-ray events were reweighed according to experimental measurements of their spectra, following the same procedure adopted in [16]. The final analysis cuts were based on the background rejection, shower arrival direction, and event multiplicity parameters. They were defined, in each considered energy bin and off-axis bin, by optimizing the sensitivity for a given exposure time. Then, five standard deviations ( $5 \sigma$, with $\sigma$ defined as in Eq. 17 of [23]) were required for a detection in each energy bin and off-axis bin, considering the same exposure time (as in the cut optimization procedure) and a ratio of the offsource to on-source exposure equal to 5. In addition, the signal excess was required to be larger than 10 and at least 5 times the expected systematic uncertainty in the background estimation (assumed to be $\sim 1 \%$ ). All of these assumptions are commonly adopted in the IACT community (see e.g. $[5,16]$ ) and allow us to derive performance results under coherent analysis conditions.

\section{Performance}

The main metrics considered to characterize the ASTRI MA performance were the differential flux sensitivity (for five different exposure times), energy resolution, angular resolution, gammaray effective area, and residual background rate for observations of point-like gamma-ray sources in dark sky conditions at a zenith angle of $20^{\circ}$. All of these quantities were comprehensively evaluated for both on- and off-axis source observations in the (reconstructed) energy range between $10^{-0.5} \simeq 0.3 \mathrm{TeV}$ and $10^{2.5} \simeq 300 \mathrm{TeV}$. Next, we present the most relevant results. 
Fig. 2 shows the on-axis point-like source differential sensitivity of the ASTRI MA for five exposure times: 0.5 (dark gray), 5 (red), 50 (blue), 200 (green), and 500 (orange) hours. The two longest exposure times were considered in order to provide sensitivity curves for deep ( 200 hours) and very deep ( $~ 500$ hours) observations, as foreseen in the ASTRI MA core science program [10]. The differential sensitivities of other instruments in the Northern Hemisphere [5, 6, 12, 13, 24] are shown for comparison. In the case of the CTAO Northern Array (CTAO-N), in addition to the configuration to be built in the construction phase (Alpha, 2028) [5] - composed of 4 large-sized telescopes (LSTs) and 9 medium-sized telescopes (MSTs) - , an earlier configuration foreseen for the science verification phase $(S V-N 3,2025)$ [24] - composed of 4 LSTs and 5 MSTs - is also considered in order to better put into context the different commissioning time scale between instruments.

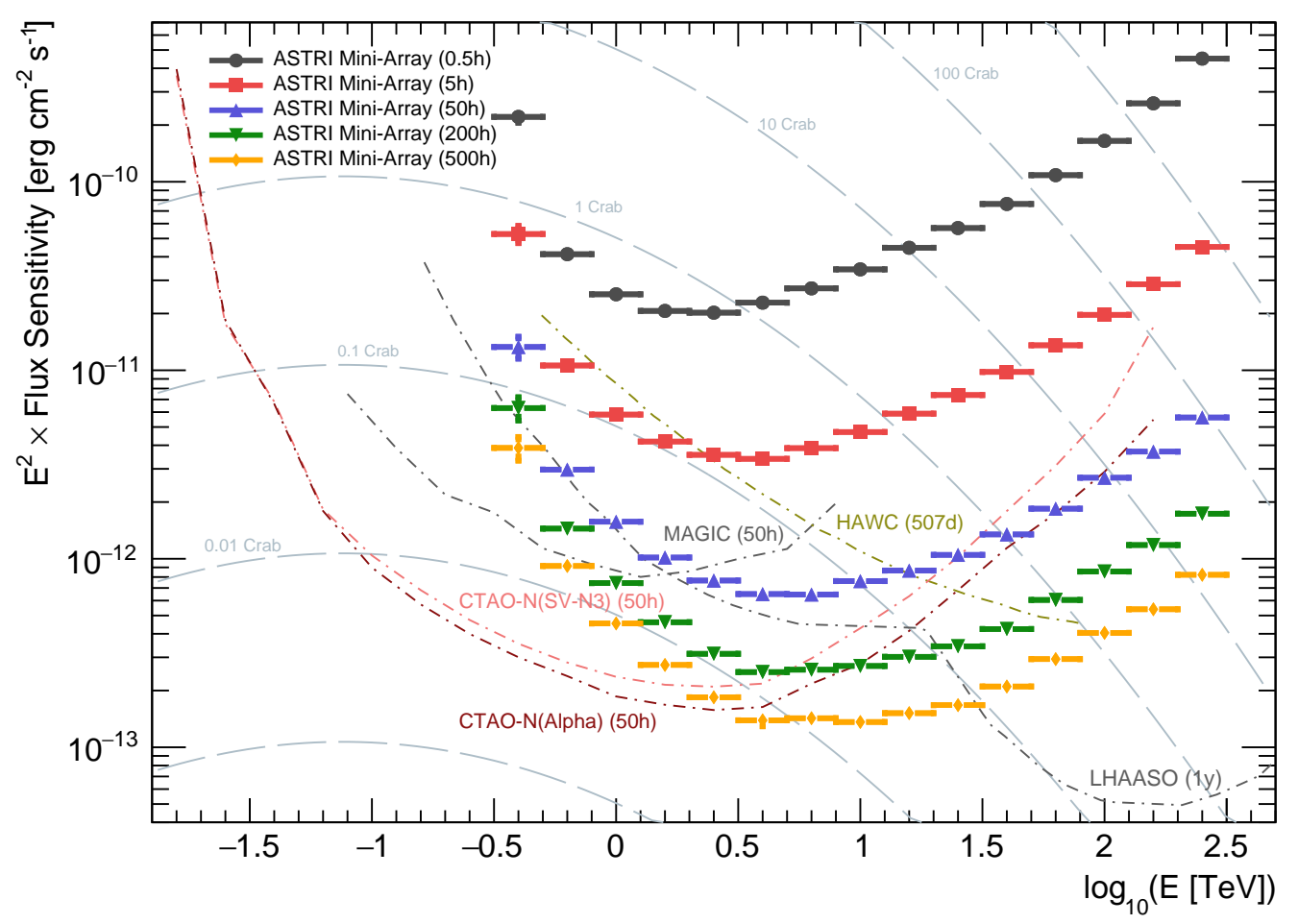

Figure 2: On-axis point-like source differential sensitivity of the ASTRI MA (at a zenith angle of $20^{\circ}$ ) for five exposure times: 0.5 (dark gray), 5 (red), 50 (blue), 200 (green), and 500 (orange) hours. The differential sensitivities of other instruments - MAGIC (50 hours) [12], HAWC (507 days) [13], LHAASO (1 year) [6], and CTAO-N (50 hours), for both science verification phase (SV-N3, 2025) [24] and construction phase (Alpha, 2028) [5] - are shown for comparison. The Crab Nebula spectral model is taken from [25]. See the main text for details on the actual procedure adopted for the calculation of the differential sensitivity.

Fig 3 shows the on-axis angular resolution (left) and energy resolution (right). The angular resolution is defined as the $68 \%$ gamma-ray event containment radius (in degrees), while the energy resolution is derived in each (reconstructed) energy bin from a Gaussian fit around the peak of the distribution of reconstructed energy over true energy. All (dark gray) curves of Fig. 3 were evaluated with analysis cuts optimized for differential sensitivity in 50 hours. It should be noted that these 
cuts do not provide the best angular and energy resolution achievable by the system. Other analysis cuts, which take into account both differential flux sensitivity and angular/energy resolution in the cut optimization process, may actually provide better performance (dashed dark green lines).
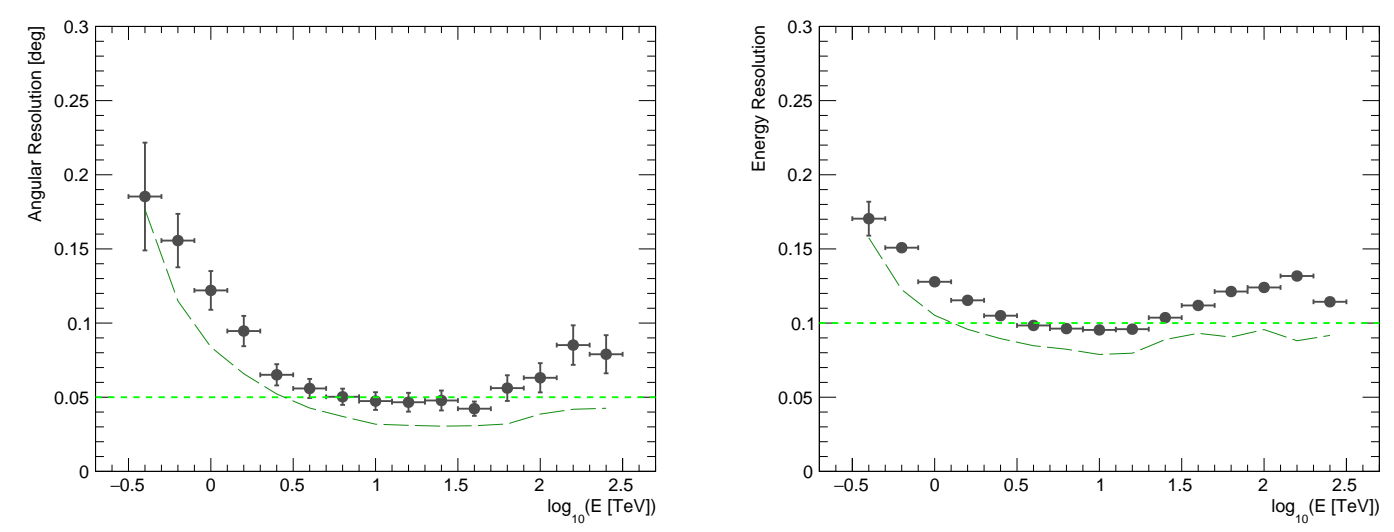

Figure 3: On-axis angular resolution (left) and energy resolution (right) of the ASTRI MA (at a zenith angle of $20^{\circ}$ ). All dark gray curves were achieved with analysis cuts optimized for differential sensitivity in 50 hours. The dashed dark green line in the left(right) panel shows the angular(energy) resolution achieved with cuts that take into account both differential sensitivity and angular(energy) resolution in the cut optimization process, while the dashed light green horizontal line represents an angular(energy) resolution of $3 \operatorname{arcmin}(10 \%)$.

Fig. 4 (left panel) shows the off-axis point-like source differential sensitivity of the ASTRI MA in 50 hours for five off-axis bins between $0^{\circ}$ and $5^{\circ}$. In the right panel of Fig. 4, the ratio between each off-axis sensitivity and that achieved in the first off-axis bin is also shown ${ }^{2}$.
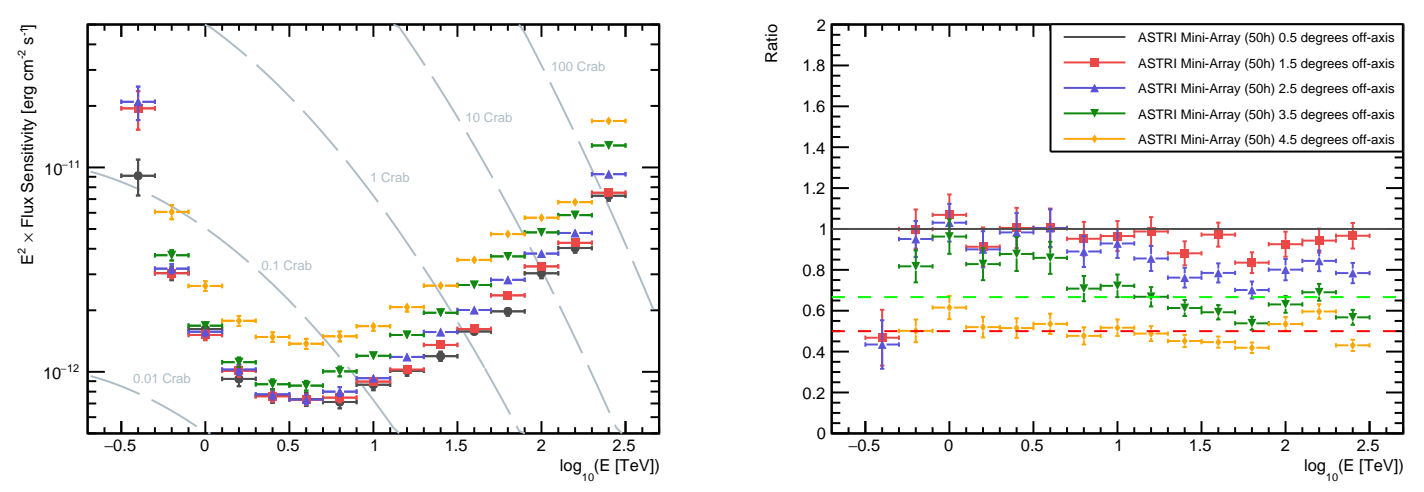

Figure 4: Left: Off-axis point-like source differential sensitivity of the ASTRI MA in 50 hours (at a zenith angle of $20^{\circ}$ ) for five off-axis bins between $0^{\circ}$ and $5^{\circ}$. The Crab Nebula spectral model is taken from [25]. See the main text for details on the actual procedure adopted for the calculation of the differential sensitivity. Right: Ratio between each off-axis sensitivity and that achieved in the first off-axis bin. The ratios are calculated so that higher values correspond to better performance. The dashed green and red lines represent a decrease in performance by a factor 1.5 and 2 , respectively.

${ }^{2}$ Although not shown in this contribution, similar ratios were found also for the off-axis angular/energy resolution. 
The key performance features of the ASTRI MA that can be derived from Fig. 2, 3, and 4 can be summarized as follows:

- The ASTRI MA will efficiently cover the TeV and multi-TeV energy band (up to $100 \mathrm{TeV}$ and beyond) with a sensitivity better than present-generation IACTs [11, 12] above a few $\mathrm{TeV}$. Furthermore, the sensitivity is expected to be comparable to that of the CTAO-N in the construction phase configuration (Alpha, 2028) [5] above a few tens of TeV. In the higher part of the energy band HAWC (507 days) [13] and, particularly, LHAASO (1 year) [6] show a significant better sensitivity, although with a substantially worse angular resolution than IACTs. All of these features will make the ASTRI MA a fully functional complement to both present- and next-generation gamma-ray observatories in the Northern Hemisphere.

- The ASTRI MA will achieve an angular and energy resolution of a few arcmin and $\sim 10 \%$ above a few $\mathrm{TeV}$, respectively. These features will enable unprecedented morphological and spectral studies of extended gamma-ray sources at the $\mathrm{TeV}$ and multi-TeV energy band. In particular, it will be possible to significantly improve the spatial characterization of sources detected above tens of TeV by HAWC [26] and LHAASO [27].

- The ASTRI MA will have an excellent off-axis performance over a wide field of view (FoV) of several squared degrees: the performance up to $3^{\circ}\left(5^{\circ}\right)$ from the center of the FoV will be within a factor of $\sim 1.5(\sim 2)$ equal to the nominal on-axis performance in the entire energy range of the system. This will allow the simultaneous observation of multiple gamma-ray sources in crowded regions and large surveys of the sky [10], enhancing at the same time the chance for serendipitous discoveries.

\section{Conclusions}

The ASTRI Mini-Array is a next-generation ground-based gamma-ray observatory under construction at the Observatorio del Teide. The full array will be operational within the next few years. In this contribution we presented the performance of the system obtained with detailed high-statistic Monte Carlo simulations and suitable analysis tools. The achieved results demonstrate the significant capabilities of the instrument for observations in the $\mathrm{TeV}$ and multi-TeV energy band, particularly important for simultaneous and follow-up observations with other present- and next-generation gamma-ray observatories located in the Northern Hemisphere.

The performance presented in this contribution was achieved considering observations in dark sky conditions at low zenith angles. Further dedicated MC productions are being produced in order to investigate the ASTRI MA performance under various observation conditions, such as higher zenith angles, higher levels of the night sky background, and possible different telescope pointing configurations. In addition to this, the data reduction and analysis software is being further developed in order to implement and test more sophisticated and precise event reconstruction methods. Some improvement of the data analysis performance is also expected.

\section{Acknowledgments}

This work was conducted in the context of the ASTRI Project. This work is supported by the Italian Ministry of University and Research (MUR) with funds specifically assigned to the Italian National Institute for Astrophysics 
(INAF). We acknowledge support from the Brazilian Funding Agency FAPESP (Grant 2013/10559-5) and from the South African Department of Science and Technology through Funding Agreement 0227/2014 for the South African Gamma-Ray Astronomy Programme. This work has been supported by H2020-ASTERICS, a project funded by the European Commission Framework Programme Horizon 2020 Research and Innovation action under grant agreement n. 653477. IAC is supported by the Spanish Ministry of Science and Innovation (MICIU). We also thank the Fundación Galileo Galilei - INAF, Fundación Canaria (FGG) for its support in deploying and managing the ASTRI Mini-Array in Tenerife.

\section{References}

[1] Aharonian, F., Bergström, L. and Dermer, C., Astrophysics at Very High Energies, Springer (2013).

[2] de Naurois, M., \& Mazin, D., Comptes Rendus Physique, 16(6-7), 610 (2015).

[3] Knödlseder, J., Comptes Rendus Physique, 17(6), 663 (2016).

[4] Acharya, B. S., et al., Science with the Cherenkov Telescope Array, World Scientific Press (2017).

[5] Gueta, O., Proc. 37th ICRC, Berlin, PoS(ICRC2021)885 (2021).

[6] Cao, Z., Proc. 37th ICRC, Berlin, PoS(ICRC2021)011 (2021).

[7] Barres de Almeida, U., Proc. 37th ICRC, Berlin, PoS(ICRC2021)893 (2021).

[8] Antonelli, L. A., Proc. 37th ICRC, Berlin, PoS(ICRC2021)897 (2021).

[9] Lombardi, S., et al., A\&A, 634, A22 (2020).

[10] Vercellone, S., Proc. 37th ICRC, Berlin, PoS(ICRC2021)896 (2021).

[11] Park, N., et al., Proc. 34th ICRC, The Hague, PoS(ICRC2015)771 (2015).

[12] Aleksić, J., et al., Astropart. Phys., 72, 76 (2016).

[13] Abeysekara, A. U., et al., ApJ, 843, 116 (2017).

[14] Heck, D., et al., Report FZKA, 6019, Forschungszentrum Karlsruhe (1998).

[15] Bernlöhr, K., Astropart. Phys., 30, 149 (2008).

[16] Acharya, B. S., et al., Astropart. Phys., 111, 35 (2019).

[17] Arrabito L., et al., Journal of Physics: Conference Series 664, 032001 (2015).

[18] Puerto-Gimenéz, I., et al., Proc. 33th ICRC, Rio de Janeiro, ID0609 (2013).

[19] Lombardi, S., et al., Proc. SPIE, 10707, 107070R (2018).

[20] Knödlseder, J., et al., A\&A, 593, A1 (2016).

[21] Deil, C., et al., Proc. 35th ICRC, Busan, PoS(ICRC2017)766 (2017).

[22] Breiman, L., Machine Learning, 45(1), 5-32 (2001).

[23] Li, T.-P. and Ma, Y.-Q., ApJ, 272, 317 (1983).

[24] Zanin, R. (CTAO Project Scientist), private communication (2021).

[25] Albert, J., et al., ApJ, 674, 1037 (2008).

[26] Albert, A., et al., ApJ, 905, 76 (2020).

[27] Cao, Z., et al., Nature, 594, 33-36 (2021). 